\title{
Structure and Damping of Toroidal Drift Waves (and their Implications for Anomalous Transport)
}

\author{
J.B. Taylor \\ Institute for Fusion Studies \\ The University of Texas at Austin \\ Austin, Texas 78712 \\ and \\ J. Connor and H.R. Wilson \\ AEA Fusion \\ Culham Laboratory \\ Abingdon OX14 3DB \\ United Kingdom (Euratom/UKAEA Fusion Association)
}

\begin{abstract}
The conventional theory of high-n toroidal drift waves, based on the ballooning representation, indicates that shear-damping is generally reduced in a torus compared to its plane-slab value. It therefore describes the most unstable class of toroidal drift waves. However, modes of this type occur only if the diamagnetic frequency $\omega^{*}(r)$ has a maximum in $r$, and they affect only a small fraction, $\mathcal{O}\left(1 / n^{1 / 2}\right)$, of the plasma radius around this maximum. Consequently they may produce little anomalous transport.

In the present work we show that, within the ballooning description, there is another class of toroidal drift waves with very different properties to the conventional ones. The new modes have greater shear-damping (closer to that in a plane-slab) than the conventional ones and so have a higher instability threshold. However, they occur
\end{abstract}


for any plasma profile and at all radii, and they have larger radial extent. Consequently they may produce much greater anomalous transport than the possibly benign conventional modes.

This suggests a picture of anomalous transport in which the plasma profile is determined by marginal stability, but marginal to the new class of modes not to the conventional ones. This might explain why marginally stable profiles calculated for drift waves with plane-slab damping sometimes agree well with the profiles in toroidal experiments. It is also consistent with the fact that experimental profiles may exceed conventional toroidal instability thresholds. The new modes may also be related to the long radial structures which appear in some plasma simulations and in experiments. 


\section{Introduction}

Several potential instabilities of magnetically confined plasmas are associated with electrostatic drift waves. Such waves, which have short wavelength across the magnetic field, long wavelength parallel to it, and frequency close to the electron diamagnetic frequency $\omega^{*}(r)$, have been extensively studied. However, we will show that there is a class of toroidal drift waves which have hitherto been overlooked and which may be important for the interpretation of anomalous transport.

In a cylinder, as in a plane slab, drift waves experience shear-damping. ${ }^{16} \mathrm{~A}$ mode $\sim \exp i(n z / L-m \theta)$ is centered on the resonant surface $m-n q(r)=0\left(q(r) \equiv r B_{z} / L B_{\theta}\right)$ and the damping is due to the energy 'radiated' from the resonant surface and eventually lost in ion Landau damping or other dissipative processes. This shear-damping may stabilize drift modes in a cylinder. It was first shown by Taylor ${ }^{18}$ that in a toroidal system, coupling between terms of different $m$ could lead to drift modes in which the shear-damping was suppressed. This was confirmed ${ }^{10,1}$ by extensive calculations using the 'Ballooning representation' $2,9,13$

The ballooning representation provides a transformation from the periodic domain $0 \leq$ $\theta<2 \pi$ to an infinite domain $-\infty<\eta<\infty$ with the periodicity condition replaced by conditions on the behaviour at infinity. This allows one to exploit the existence of two disparate radial scale lengths at high toroidal mode number - the equilibrium scale length $r$ and the separation of mode resonant surfaces $1 / n q^{\prime}(r)$. The perturbation can be expressed as

$$
\phi=\mathcal{E}(q, \eta) \exp i[n \zeta-n q(\eta-\kappa)-\Omega t]
$$

where $\kappa$ is a free parameter which locates the mode in poloidal angle, $q(r)$ is used as radial coordinate and $\zeta$ is the toroidal angle. Then an expansion in the small parameter $1 / n$ 
leads in lowest order to a differential equation for $\mathcal{E}$ in $\eta$ alone, with the other coordinate $q$ appearing only as a parameter. The condition that $\mathcal{E}$ be well behaved at infinity leads to a local eigenvalue $\omega(q, \kappa)$.

The quantity $\omega(q, \kappa)$ itself is often regarded as the frequency of a toroidal drift wave, but it is important to recognize that the calculation described so far does not, in fact, determine a global eigenfrequency. To do so requires consideration of higher order terms in the $1 / n$ expansion. This shows that if the function $\omega(q, \kappa)$ can be expanded about a stationary point, where $\omega\left(q_{0}, \kappa_{0}\right)=\omega_{0}$, then a toroidal drift mode indeed exists with a frequency close to $\omega_{0}$. This is the mode whose damping is suppressed relative to that in a cylinder.

However, if the damping is small compared to the real frequency, as is implicit in most calculations, yo must be near a maximum in the diamagnetic frequency $\omega^{*}(q)$. Consequently, the conventional toroidal drift modes can exist only where there is a maximum in $\omega^{*}(q)$. In the next section we show that, within the ballooning theory, there is another class of drift waves which are not related to a maximum in $\omega^{*}(q)$.

\section{A Model Calculation}

A model ${ }^{4}$ which describes drift waves in a torus with inverse aspect ratio $\epsilon_{n} \ll 1$ and $k a_{i} \ll 1$ (where $k=n q / r$ is the poloidal wavenumber and $a_{i}$ is the ion Larmor radius) is defined by the equation

$$
\begin{aligned}
& {\left[\left(\frac{1}{\left(n q^{\prime}\right)^{2}} \frac{\partial^{2}}{\partial x^{2}}-\frac{1}{s^{2}}\right)-\sigma^{2}\left(\frac{\partial}{\partial \theta}+i n q^{\prime} x\right)^{2}-\epsilon\left(\cos \theta+\frac{i s}{n q^{\prime}} \sin \theta \frac{\partial}{\partial x}\right)\right.} \\
& \left.+\left(\Omega^{*}(x)-\Omega\right)\right] \phi(x, \theta)=0
\end{aligned}
$$

where $x$ is distance from some rational surface and $s$ is the shear $r q^{\prime} / q$. The first term in Eq. (2) arises from finite Larmor radius and the second from ion sound (these terms lead to shear damping in a cylinder). The third term is the effect of toroidal coupling. In Eq. (2), $1 / n$ 
is a small parameter but $\sigma=\epsilon_{n} / k^{2} a_{i}^{2} q s, \epsilon=2 \epsilon_{n} / k^{2} a_{i}^{2} s^{2}$ and $\left(\Omega^{*}-\Omega\right)=\left(\omega^{*}-\omega\right) / \omega_{0}^{*} k^{2} a_{i}^{2} s^{2}$ are of order unity.

We write the perturbation, using the ballooning transformation, as

$$
\phi(x, \theta)=\sum_{m} e^{-i m \theta} \int e^{+i m \eta} \hat{\phi}(x, \eta) d \eta
$$

then $\hat{\phi}$ satisfies Eq. (2) in the extended domain $-\infty<\eta<\infty$ and need not be periodic. The removal of the periodicity constraint allows us to write $\hat{\phi}$ in an eikonal, or WKB, form. In order to combine discussion of the new and of the conventional modes, it is convenient to use

$$
\widehat{\phi}(x, \eta)=\xi(x, \eta) \exp \left[-i n q^{\prime}(x \eta-S(x))\right]
$$

instead of Eq. (1).

Then, to lowest order in $1 / n, \mathcal{E}$ must satisfy an ordinary differential equation in $\eta$ at each $x$,

$$
\left[\sigma^{2} \frac{d^{2}}{d \eta^{2}}+\frac{1}{s^{2}}+(\eta-k)^{2}+\epsilon(\cos \eta+s(\eta-k) \sin \eta)-\left(\Omega^{*}(x)-\Omega\right)\right] \xi_{0}(k, \eta)=0
$$

where $k \equiv d S / d x$. The eigenvalue for this equation defines a function $\Omega(x, k)$ periodic in $k$. In the present simple model $\Omega(x, k)=\Omega^{*}(x)+g(k)$ where $g(k)$ is periodic and incorporates the shear damping. Note also that $\xi(\eta+2 \pi, k+2 \pi)=\xi(\eta, k)$.

We consider first the conventional drift modes. These arise when the diamagnetic frequency has a maximum at some point $x_{0}$. Then we write

$$
\Omega=\Omega_{0}+\frac{1}{2}\left[\left(x-x_{0}\right)^{2} \Omega_{x x}^{*}+\left(k-k_{0}\right)^{2} g_{k k}\right]
$$

where $k_{0}$ is a stationary point of $g(k)$ corresponding to minimum damping. Eq. (6) defines two branches $k^{+}(x, \Omega)$ and $k^{-}(x, \Omega)$ that provide WKB solutions

$$
\alpha \exp \left(i n q^{\prime} \int^{x} k^{+} d x^{\prime}\right), \quad \beta \exp \left(i n q^{\prime} \int^{x} k^{-} d x^{\prime}\right)
$$

valid except near the turning points where $k^{+}=k^{-}, d k^{ \pm} / d x=\infty$. 
To construct a global solution one selects the WKB forms which decay as $x \rightarrow \pm \infty$ and extends each around the appropriate left or right turning point. Matching the two forms then leads to the usual WKB eigenvalue condition

$$
\frac{n q^{\prime}}{2 \pi} \int\left(k^{+}-k^{-}\right) d x^{\prime}=\text { integer }+\frac{1}{2}
$$

This construction is illustrated schematically in Fig. 1. For comparison with the later construction, note that a single 'external' branch from $+\infty$ connects to two 'internal' branches at the turning point $x_{R}$, and that the same two branches are connected to a single external branch from $-\infty$ at the other turning point $x_{L}$. The integral in Eq. (8) corresponds to the shaded area within the internal loop.

As we have noted, modes constructed in this way occur only near a maximum in $\Omega^{*}(x)$. Furthermore, although they extend over many resonant surfaces, they affect only a fraction $\mathcal{O}\left(1 / n^{1 / 2}\right)$ of the plasma radius. Because their wavenumber $k(x)$ is restricted to a small range around $k_{0}$, corresponding to minimum damping, the damping of these modes is 'suppressed' relative to that in a cylinder.

We now turn to the consideration of the other class of toroidal modes. These do not recuire a maximum in $\Omega^{*}$ and can be described by treating $\Omega^{*}(x)$ as locally linear in $x$, $\Omega^{*}=\Omega_{0}+\lambda x$. Then for the local eigenvalue, instead of Eq. (6), we have

$$
\Omega(x, k)=\Omega_{0}+\lambda x+g(k) .
$$

The construction of a WKB solution is now quite different from the previous case. We must recognize that $k(x)$ has an infinite number of (periodic) branches and that it is no longer possible to construct a solution which involves only a single branch at $\pm \infty$. The problem is illustrated schematically in Fig. 2. If one considers a single external branch $k_{\bar{N}}^{-}$from $+\infty$, this connects to the two internal branches $k_{N}^{+}$and $k_{N}^{-}$at $x_{R}$, but these branches do not reconnect at the left turning point. Instead they connect to the external branches $k_{N}^{+}$and 
$k_{N-1}^{+}$and also to two internal branches $k_{N+1}^{-}$and $k_{N-1}^{+}$. Thus any single WKB branch is eventually connected to every other branch via the 'ladder' of internal branches.

To construct a global solution, therefore, one must include all the branches from $\pm \infty$. For example, a solution for $x>x_{R}$ is

$$
f^{+}=\sum f_{N}^{+}=\sum \alpha_{N} \xi\left(k_{N}^{-}, \eta\right) \exp \left(i n q^{\prime} \int_{x_{R}}^{x} k_{N}^{-} d x^{\prime}\right) .
$$

As already noted, at the right turning point, the external $k_{N}^{-}$connects to the internal $k_{N}^{+}$ and $k_{N}$ and for $x_{L}<x<x_{R}$ this solution becomes

$$
f^{+} \rightarrow \sum \alpha_{N}\left[\xi\left(k_{N}^{+}, \eta\right) \exp \left(i n q^{\prime} \int_{x_{R}}^{x} k_{N}^{+} d x^{\prime}\right)+\xi\left(k_{N}^{-}, \eta\right) \exp \left(i n q^{\prime} \int_{x_{R}}^{x} k_{N}^{-} d x^{\prime}\right)\right] .
$$

Similarly, a solution for $x<x_{L}$ is

$$
f^{-}=\sum f_{N}^{-}=\sum \beta_{N} \xi\left(k_{N}^{+}, \eta\right) \exp \left(i n q^{\prime} \int_{x_{L}}^{x} k_{N}^{+} d x^{\prime}\right) .
$$

At the left turning point the external $k_{N}^{+}$connects to the internal $k_{N}^{+}$and $k_{N+1}$ and for $x_{L}<x<x_{R}$ this solution becomes

$$
f^{-} \rightarrow \sum \beta_{N}\left[\xi\left(k_{N}^{+}, \eta\right) \exp \left(i n q^{\prime} \int_{x_{L}}^{x} k_{N}^{+} d x^{\prime}\right)+\xi\left(k_{N+1}^{-}, \eta\right) \exp \left(i n q^{\prime} \int_{x_{L}}^{x} k_{N+1}^{-} d x^{\prime}\right)\right] .
$$

Matching the expressions (11) and (13) in the region $x_{L}<x<x_{R}$ imposes two conditions:

$$
\alpha_{N}=\beta_{N} \exp \left(i n q^{\prime} \int_{x_{L}}^{x_{R}} k_{N}^{+} d x^{\prime}\right)=\beta_{N-1} \exp \left(i n q^{\prime} \int_{x_{L}}^{x_{R}} k_{N} d x^{\prime}\right) .
$$

At this point we express all $k_{N}^{ \pm}$in terms of the $k_{0}^{ \pm}$which lie in the basic interval $-\pi, \pi$, i.e. $k_{N}^{ \pm}=k_{0}^{ \pm}+2 \pi N$, and consider the perturbation $\hat{\phi}$ which corresponds to the solutions (10)-(13). Corresponding to Eq. (12), for example, the perturbation will have the form

$$
\hat{\phi}=\sum_{N} \beta_{N} \xi\left(k_{0}^{+}+2 \pi N, \eta\right) \exp \left[i n q^{\prime}\left(\int_{x_{L}}^{x} k_{0}^{+} d x^{\prime}-x \eta+2 \pi N\left(x-x_{L}\right)\right)\right]
$$

Recalling that $\xi(k+2 \pi, \eta)=\xi(k, \eta-2 \pi)$, we see that this will be periodic in $\eta$ if

$$
\beta_{N} \exp \left(-2 \pi i n q^{\prime} N x_{L}\right)=\text { constant } .
$$


Hence if this condition is satisfied, Eq. (15) will directly describe a periodic mode and the ballooning transformation is not needed! In fact Eq. (15) could not be used in the transformation as the resulting integral would not converge. Thus we see that the eikonal approximation may lead either to a solution which can be used in the ballooning representation (as for the conventional drift modes) or to a periodic solution which neither needs nor is suitable for the ballooning transformation (as for the present modes). The two cases are complementary. This is in accord with the observation of Dewar and Glasser ${ }^{7}$ that the ballooning transformation may be regarded simply as a device to introduce an infinite covering space for $\theta$.

Combining (14) and (16) leads to the eigenvalue condition

$$
n q^{\prime}\left[\int_{x_{L}}^{x_{R}}\left(k_{0}^{+}-k_{0}^{-}\right) d x^{\prime}+2 \pi x_{L}\right]=2 \pi \times \text { integer }
$$

in which the integral corresponds to the shaded area between the $\Omega(x, k)=$ constant curve and the $x$-axis over one period of $k$. Using Eq. (9) it may also be expressed as

$$
\frac{n q^{\prime}}{2 \pi \lambda} \int_{0}^{2 \pi}\left(g(p)+\Omega_{0}-\Omega\right) d p=\text { integer . }
$$

(This form can be derived more directly ${ }^{5}$ using a wavenumber version of the ballooning representation, but this does not bring out the connection with the conventional modes.)

At first sight the eigenvalue condition (17) is surprising, since the area it represents depends on the arbitrary origin of the $x$-coordinate! The interpretation is that there are eigenmodes with frequencies corresponding to all values of $\Omega^{*}(x)$ and the eigenvalue condition relates the frequency of any mode to its location in $x$. In the present simple model, where $\lambda$ and $\Omega_{0}$ are real, all such modes have the same damping, given by

$$
\operatorname{Im} \Omega=\frac{1}{2 \pi} \operatorname{Im} \oint g(p) d p .
$$

These modes are entirely different to the ones described earlier. Most important, of course, is the fact that they occur for all profiles, not just those with a maximum in $\Omega^{*}$, and at 
all radii. In addition, they extend over a fraction $\mathcal{O}\left(\epsilon_{n}\right)$ of the plasma radius instead of a fraction $\mathcal{O}\left(1 / n^{1 / 2}\right)$. Their radial wavenumber is not restricted to that for minimum damping so the damping of these modes is an average over $k$ and thus closer to that in a cylinder. (For example, if one expanded in $\epsilon$, the damping of the conventional modes is reduced by an amount $\mathcal{O}(\epsilon)$ compared to that in a cylinder, whereas the damping of the present modes is reduced only by an amount $\mathcal{O}\left(\epsilon^{2}\right)$.)

Although the perturbations described here are genuine eigenmodes, it is interesting to interpret them in terms of the behavior of local disturbances. For this we first recall that $k$ determines the poloidal angle at which the disturbance 'balloons' in the minor cross-section. ( $k=0$ signifying the outer edge of the torus and $k=\pi$ the inner edge). We also discard the simple model with $\Omega=\Omega^{*}(x)+g(k)$, and revert to the general form $\Omega(x, k)$ - but neglect damping so that $\Omega$ is essentially real. Then a local disturbance propagates at the group velocity, ${ }^{20}$

$$
\frac{d x}{d t}=\frac{\partial \Omega}{\partial k}
$$

and at the same time

$$
\frac{d k}{d t}=-\frac{\partial \Omega}{\partial x}
$$

so that the frequency remains constant. Thus the disturbance moves along the path of fixed $\Omega(x, k)$ (see Fig. 2), oscillating in 'radius' $(x)$ between $x_{L}$ and $x_{R}$ while rotating around the minor cross-section. Hence it experiences the average ballooning effect - leading to the averaged damping given by Eq. (19). Of course, the time required for this averaging to be effective depends on the shear $\partial \Omega / \partial x$. This behavior is similar to that of ballooning perturbations in rotating plasmas ${ }^{6,19}$ and of the radially propagating structures described by Garbet, et al.. ${ }^{8}$ The eigenmode calculated here can be regarded as the smoothed, or phase mixed, remnant of such an initial disturbance. Similarly the conventional mode can 
be regarded as the remnant of an initial disturbance trapped at the stationary point of $\Omega(x, k)$. Equations $(20)$ and $(21)$ may also describe purely convective modes.

\section{Discussion}

We have emphasized that the conventional calculation of high mode number toroidal drift waves describes modes that exist only when the diamagnetic frequency has a maximum, and that they affect only the vicinity of this maximum. Their shear damping may be significantly suppressed by toroidal coupling compared to that of modes in a cylinder.

We have also shown that there is another class of high mode number toroidal drift waves which have markedly different characteristics. These modes occur at all plasma radii, not just at maxima of the diamagnetic frequency, and when $\epsilon_{n}>1 / n^{1 / 2}$ they extend over a larger fraction of the plasma radius. Their shear damping is less affected by toroidal coupling and consequently closer to that in a cylinder.

Although these results are based on a simple model of electron drift waves, similar conclusions should apply to more complex models and to other types of instability (e.g. $\eta_{i}$ and $\nabla T_{i}$ modes). They may, therefore, be important for the interpretation of anomalous transport in tokamaks (although, of course, this involves nonlinear effects whereas we have considered only linear properties).

For example, because they occur at all radii and have considerable radial extent and overlap, the new modes are likely to produce much more transport than the conventional ones - which, although they may have a lower instability threshold, affect at most only a small fraction of the radius. This suggests a picture in which the plasma profile is determined by marginal stability, but marginal to modes of the new class, not the conventional ones. In addition to the implication of 'profile consistency,' this might explain why experimental profiles sometimes exceed the threshold given by conventional toroidal instability calculations, ${ }^{11}$ but why, on the other hand, marginal stability profiles calculated using plane slab (or cylinder) 
damping sometimes agree well with experiments. ${ }^{14,15}$ The new eigenfunctions might also be related to the long radial structures recently seen in some plasma simulations. ${ }^{12,17}$

\section{Acknowledgments}

One of us (JBT), would like to acknowledge helpful discussions with M. Kotschenreuther, F. Waelbroeck, and R. Waltz.

This work was supported by the U.S. Department of Energy contract \#DE-FG05-80ET53088 , the UK Department of Trade and Industry, and Euratom. 


\section{References}

'Chen, L., and Cheng, C.Z., 1987, Physics Fluids 30, 3180.

${ }^{2}$ Connor, J.W., Hastie, R.J., and Taylor, J.B., 1978, Phys. Rev. Lett. 40, 396.

${ }^{3}$ Connor, J.W., Hastie, R.J., and Taylor, J.B., 1979, Proc. Roy. Soc. A 365, 1.

'Connor, J.W., and Taylor, J.B., 1987, Physics Fluids 30, 3180.

${ }^{5}$ Connor, J.W., Taylor, J.B., and Wilson, H.R., 1993, Phys. Rev. Lett. 70, 1803.

${ }^{6}$ Cooper, W.A., 1988, Plasma Physics and Controlled Fusion 30, 1805.

`Dewar, R.L., and Glasser, A.H., 1983, Physics Fluids 26, 3038.

${ }^{8}$ Garbet, X., Laurent, L., Roubin, J.P., and Samain, A., 1992 Proceedings IAEA Conference Würzburg, Paper D-4-4.

${ }^{9}$ Glasser, A.H., 1977, Proc. Finite Beta Theory Workshop Varenna (U.S. Dept. of Energy Report CONF-7709167) p. 55.

${ }^{10}$ Hastie, R.J., Hesketh, K.W., and Taylor, J.B., 1979, Nuclear Fusion 19, 1223.

${ }^{11}$ Kotschenreuther, M., 1993 in Ion Temperature Cradient Driven Turbulent Transport (Eds.) W. Horton, I. Wakatani, and A. Wootton, to be published by AIP.

${ }^{12}$ LeBrun, M.J., Tajima, T., Gray, M.G., Furnish, G., and Horton, W., 1993, Physics Fluids B 5, 752.

${ }^{13}$ Lee, Y.C., and Van Dam, J.W., 1977, Proc. Finite Bcta Theory Workshop Varenna (U.S. Dept. of Energy Report (CONF-7709167) p. 93. 
${ }^{14}$ Manheimer, W.M., and Antonsen, T.M., 1979, Physics Fluids 22, 957.

${ }^{15}$ Manheimer, W.M., Chu, K.R., and Boris, J.P., 1976, Phys. Rev. Lett. 37286.

${ }^{16}$ Pearlstein, L.D., and Berk, H.L., 1969, Physics Fluids 23, 220.

${ }^{17}$ Tang, W.M., and Rewoldt, G., 1993, Physics Fluids B to be published.

${ }^{18}$ Taylor, J.B., 1977 Plasma Physics and Controlled Nuclear Fusion Research vol. 2 (Internatonal Atomic Energy Agency Vienna) p. 323.

${ }^{19}$ Waelbroeck, F., and Chen, L., 1992, Physics Fluids B 3, 601.

${ }^{20}$ Weinberg, S., 1962, Phys. Rev. 126, 1899.

DISCLAIMER

This report was prepared as an account of work sponsored by an agency of the United States Government. Neither the United States Government nor any agency thereof, nor any of their employees, makes any warranty, express or implied, or assumes any legal liability or responsibility for the accuracy, completeness, or usefulness of any information, apparatus, product, or process disclosed, or represents that its use would not infringe privately owned rights. Reference herein to any specific commercial product, process, or service by trade name, trademark, manufacturer, or otherwise does not necessarily constitute or imply its endorsement, recommendation, or favoring by the United States Government or any agency thereof. The views and opinions of authors expressed herein do not necessarily state or reflect those of the United States Government or any agency thereof. 


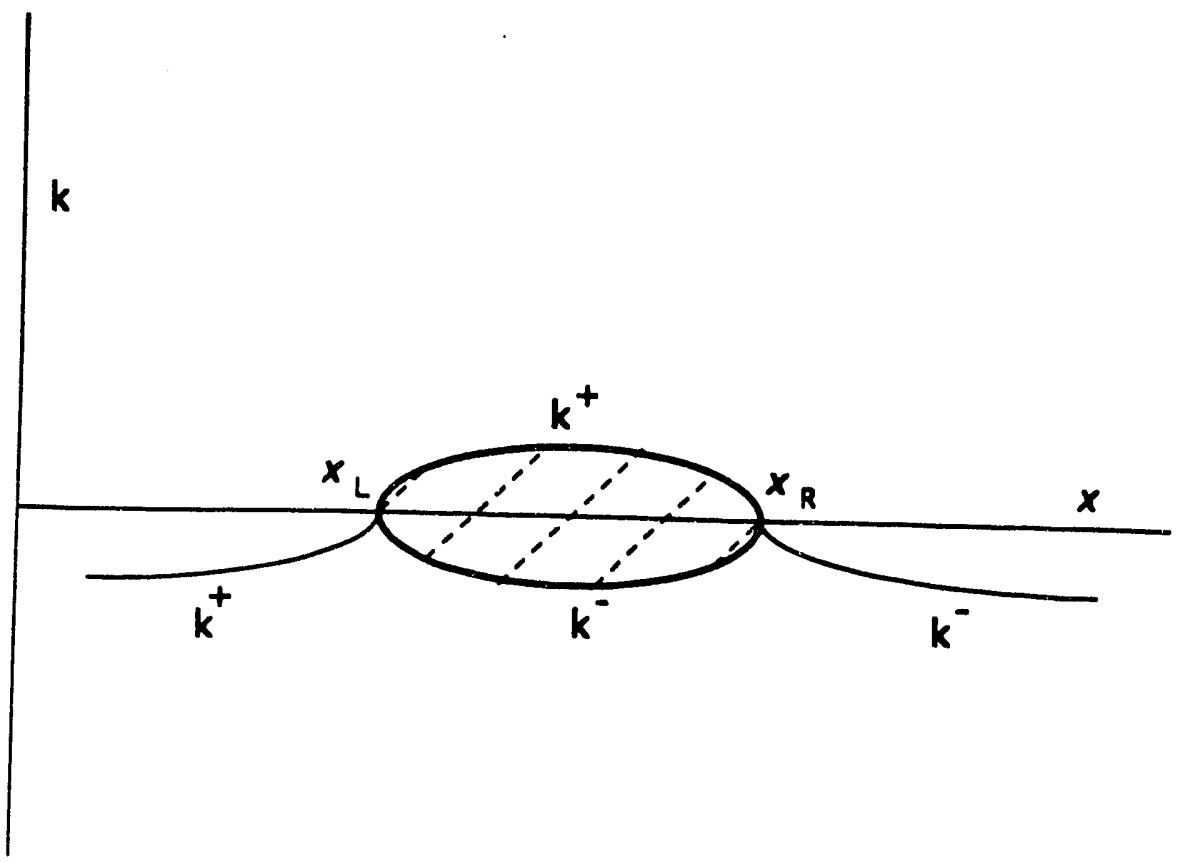

Fig. 1 Schematic WKB Branches for Eq (6).

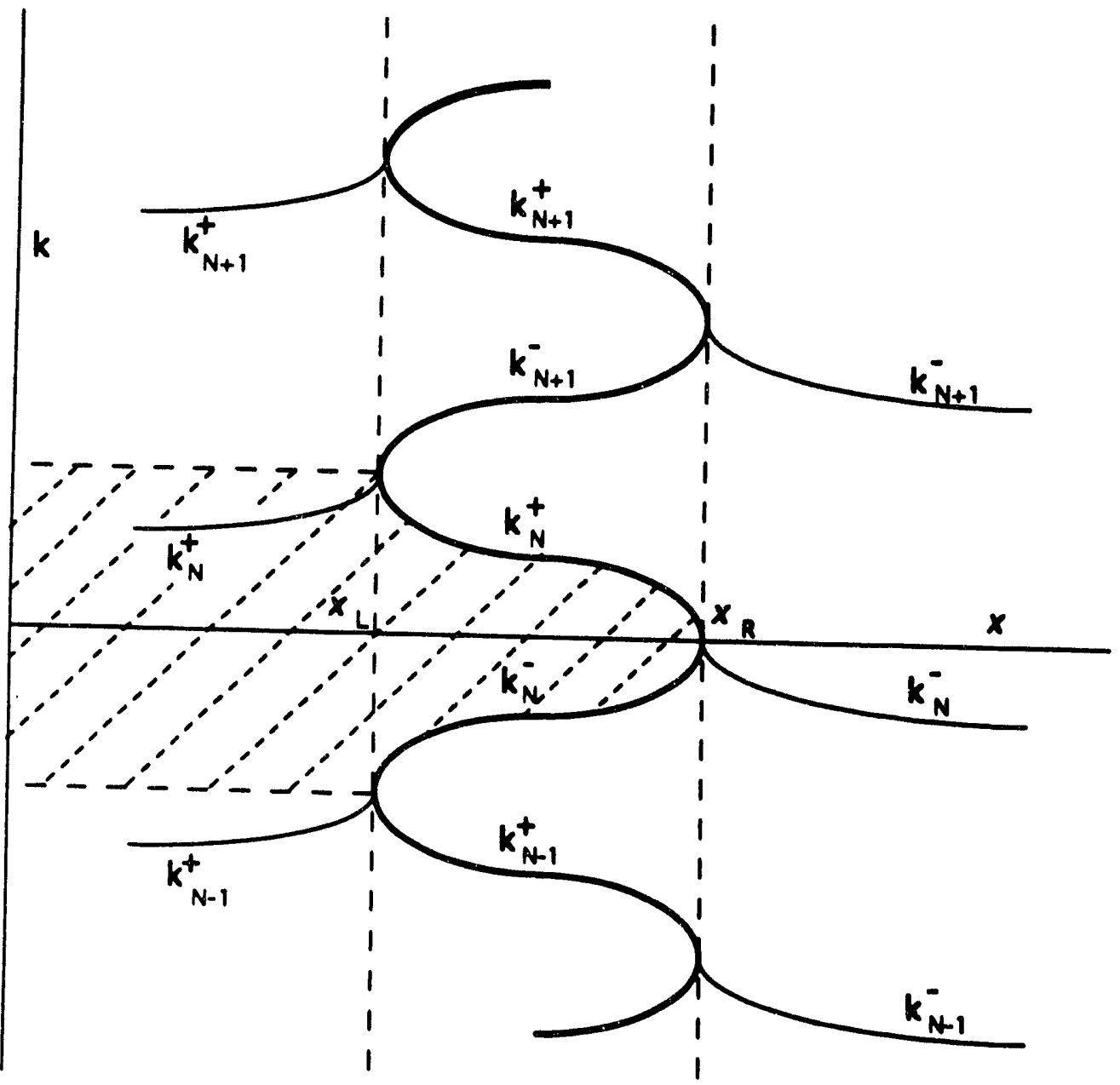

Fig 2. Schematic WKB Branches for Eq(9) 

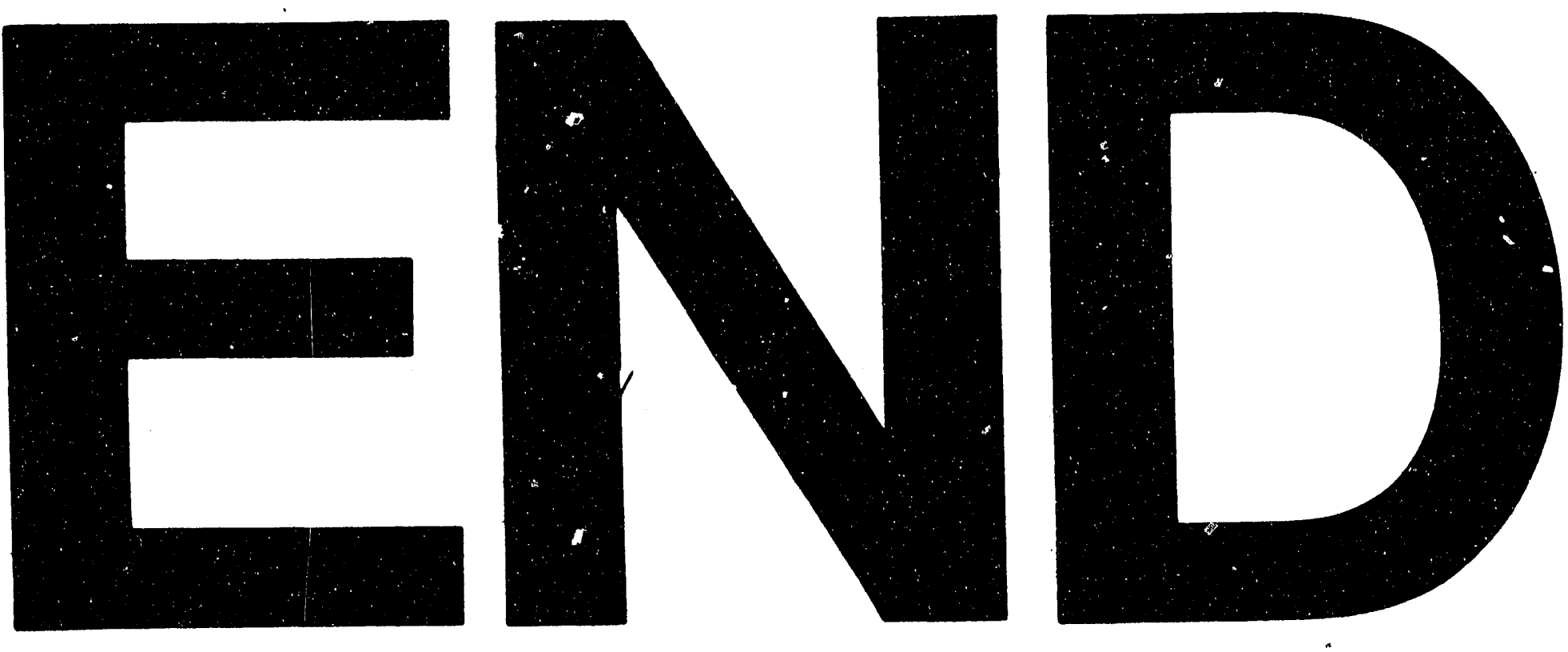

1
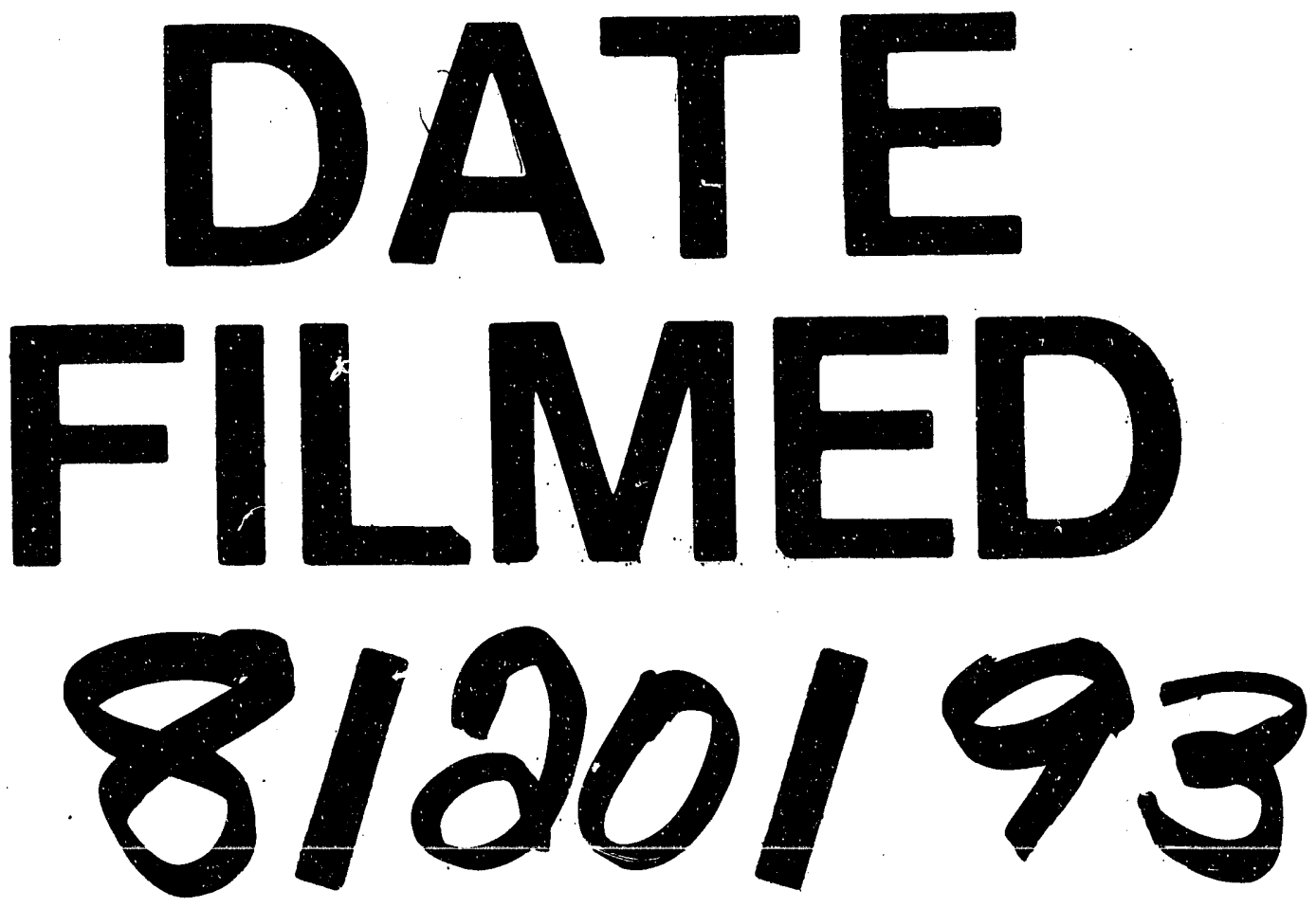
